\section{§14. On Turbulent Transport in Burning Plasmas}

Itoh, K., Yagi, M., Itoh, S.-I. (RIAM, Kyushu U.), Fukuyama, A. (Kyoto U.)

One of the key issues for the high temperature plasmas is the turbulence-driven transport of burning plasmas which are confined in toroidal magnetic confinement devices. We here study the scale invariance property [1] of the reduced set of equations in the presence of the selfheating term due to the fusion reaction.

We choose a model equation of perturbations of the static potential $\phi$, current $j$ and pressure $p$ as [2]

$\frac{d}{d t}\left(\begin{array}{c}\nabla_{\perp}^{2} \phi \\ j \\ p\end{array}\right)+\left(\begin{array}{ccc}0 & -i k_{\|} & i k_{y} G_{0} \\ i k_{\|} & 0 & 0 \\ i k_{y} & 0 & F\end{array}\right)\left(\begin{array}{c}\phi \\ j \\ p\end{array}\right)=0$

where $F=3^{-1} \partial P_{f} / \partial p$ and $\frac{d}{d t}=\frac{\partial}{\partial t}+[\phi$,$] . The$ term $F p$, i.e., the variation of the fusion energy source, is added, noting the fact that the fusion energy source is dependent on the local plasma pressure.

In order to clarify the arguments, collisional dissipation terms are neglected. In Eq.(1), the matrix includes the inhomogeneity parameter $G_{0}$ and the term $F$ which represents the fusion reaction.

Let us consider a transformation

$$
x \rightarrow \lambda_{1} x \quad \text { and } t \rightarrow \lambda_{2} t
$$

Field quantities are transformed as

$$
\left(\begin{array}{c}
\phi \\
j \\
p
\end{array}\right) \rightarrow\left(\begin{array}{l}
\lambda_{1}^{2} \lambda_{2}^{-1} \phi \\
\lambda_{1}^{2} j \\
\lambda_{1} p
\end{array}\right)
$$

In the equation of energy balance, two terms $d p / d t$ and $F p$ follow the same transformation. That is, the transformation

$$
F \rightarrow \frac{1}{\lambda_{2}} F
$$

holds. From the equation of motion (vorticity equation), a transformation

$$
G_{0} \rightarrow \frac{1}{\lambda_{2}^{2}} G_{0}
$$

is required. From Eqs.(4) and (5), we can choose an arbitrary parameter $\eta$ and a combination of parameters $G_{0}^{\eta / 2} F^{1-\eta}$ obeys the scale transformation

$$
G_{0}^{\eta / 2} F^{i-\eta} \rightarrow \frac{1}{\lambda_{2}} G_{0}^{\eta / 2} F^{1-\eta} .
$$

Case of finite $k_{\|}$is studied. In this case, the term $k_{\|} j$ remains in the equation of motion. Requiring that this term has the same scaleinvariant property as other terms, one has a relation $\lambda_{1}=1 / \lambda_{2}$ [2]. One finds that the turbulent transport coefficient, $D$, which has the dimension of $D \sim$ length $^{2}$ time $e^{-1}$, obeys the transformation relation

$$
D \rightarrow \frac{1}{\lambda_{2}^{3}} D .
$$

Combining Eqs.(8) and (10), we have the relation that the quantity

$$
\frac{D}{\left(G_{0}^{\eta / 2} F^{1-\eta}\right)^{3}}
$$

is invariant under the scale transformation. In other words, one obtains that the transport coefficient has the dependence like

$$
D \propto\left(G_{0}^{\eta / 2} F^{1-\eta}\right)^{3}
$$

This result is the generalization of the transport coefficient. In the absence of the fusion power, the dependence

$$
D \propto G_{0}^{3 / 2}
$$

has been derived.

It is shown that the turbulent transport coefficient could be different in ignited plasmas.

\section{References}

[1] Connor J W 1988 Plasma Phys. Contr. Fusion 30619.

[2] Itoh K, Itoh S-I and Fukuyama A 1999 Transport and structural formation in plasmas (IOP, England) 